\title{
Las corrientes superficiales de chorro del Chocó y el Caribe durante los eventos de El Niño y El Niño Modoki
}

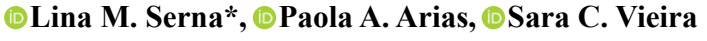 \\ Grupo de Ingeniería y Gestión Ambiental (GIGA), Facultad de Ingeniería, Universidad de Antioquia, Medellín, Colombia
}

\begin{abstract}
Resumen
El objetivo de este artículo fue analizar la influencia de dos tipos de eventos de El Niño, conocidos como Canónico y Modoki, en las corrientes superficiales de chorro del Chocó y el Caribe, importantes mecanismos de transporte de humedad y generación de precipitación en Colombia. La influencia de estos tipos de eventos de El Niño en las corrientes superficiales de interés se examinó con base en las diferencias entre las características en la climatología de estos chorros durante dichos eventos y el análisis de correlaciones entre estos fenómenos. Los resultados obtenidos indicaron que en los dos primeros trimestres del año, la intensidad de estas corrientes superficiales se ve disminuida durante ambos tipos de eventos El Niño, en tanto que en los dos últimos trimestres del año se observan efectos contrarios: un fortalecimiento del chorro del Caribe y un debilitamiento del chorro del Chocó. Sin embargo, la magnitud del impacto durante los eventos El Niño Modoki es menor que durante los eventos El Niño Canónico, aunque se observa un mayor número de eventos El Niño Modoki durante el periodo de análisis. Lo anterior es fundamental para la elaboración de pronósticos de precipitación en Colombia. (C) 2018. Acad. Colomb. Cienc. Ex. Fis. Nat.
\end{abstract}

Palabras clave: ENSO; Chorro del Chocó; Chorro del Caribe; Colombia; Modoki.

The Choco and Caribbean low-level jets during EI Niño and EI Niño Modoki events

\begin{abstract}
The objective of this paper was to analyze the influence of two types of El Niño events (known as Canonical and Modoki) on the Chocó and the Caribbean low-level jets, which are important moisture transport and precipitation generation mechanisms in Colombia. We explored the influence of these types of El Niño events on these low-level jets by analyzing the differences between climatological features of these jets and the patterns observed during the occurrence of these two El Niño events, as well as the correlations between these phenomena. Our results indicated that for the first half of the year, the strength of these low-level jets diminished during the occurrence of both types of events, while in the last half of the year opposite effects were observed, with the strengthening of the Caribbean low-level jet and the weakening of the Choco jet during the occurrence of both events. However, the magnitude of the impacts observed during Canonical El Niño events was larger than during El Niño Modoki events. This finding is essential in the development of rainfall forecasting in Colombia. (C) 2018. Acad. Colomb. Cienc. Ex. Fis. Nat.
\end{abstract}

Key words: ENSO; Choco jet; Caribbean low-level jet; Colombia; Modoki.

\section{Introducción}

La climatología de una región está determinada por una diversidad de procesos atmosféricos, así como por su posición geográfica y su geomorfología. El clima en el norte de Suramérica, y en concreto el de Colombia, por su ubicación en latitudes tropicales, registra un marcado ciclo de precipitación bimodal en la región central y en el oeste del país debido, en parte, a la migración de la zona de convergencia intertropical (ZCIT) (Poveda, 2004), a la cercanía de los océanos Pacífico y Atlántico, que facilita la llegada y el transporte de humedad atmosférica hacia el continente (Sakamoto, et al., 2012), y a la presencia de tres ramificaciones de la cordillera de los Andes y de la selva
Amazónica en su territorio y en el de los países vecinos, lo que constituye una fuente de humedad mediante procesos de evapotranspiración (Poveda, et al., 2014). Ello explica la gran diversidad de ecosistemas y biomas del país, los cuales dependen del acoplamiento y la estabilidad de estas condiciones para su supervivencia.

En las numerosas regiones que componen el territorio colombiano se puede encontrar una gran variedad de características climáticas: desde los climas secos y áridos

\footnotetext{
*Correspondencia:

Lina M. Serna; linam.sernat@gmail.com

Recibido: 23 de julio de 2018

Aceptado: 27 de octubre de 2018

Editor: José Pabón Caicedo
} 
de los desiertos, con poca o casi nula precipitación, hasta los climas con precipitaciones anuales entre 8.000 y 13.000 mm (Poveda \& Mesa, 1999) como la selva del Chocó, en la cual se registran altos índices de biodiversidad. En las zonas que se encuentran más cerca de los océanos, como es el caso del departamento de Chocó o de la costa Caribe, existe una importante influencia de estas masas oceánicas, así como de las corrientes superficiales que se generan en regiones tropicales, conocidas como corrientes superficiales de chorro, y que están estrechamente relacionadas con la proximidad de los océanos al continente y los gradientes de temperatura que se forman entre ambos. Esto propicia la circulación de corrientes de aire desde lugares con mayor presión (fríos) a lugares de menor presión (calientes). Otras condiciones que favorecen la formación, circulación e intensidad de estas corrientes en el norte de Colombia son la curvatura de los vientos alisios que, al cruzar el ecuador, son afectados por el cambio de signo de la aceleración de Coriolis, la posición de la ZCIT, los cambios en los ciclos diurnos, las oscilaciones inerciales y los cambios en la fricción de las superficies, entre otros (Prabha, et al., 2011). Estas corrientes están vinculadas al transporte y el flujo de la humedad desde los océanos hacia los continentes, al desarrollo de la actividad de convección, a la liberación de calor latente hacia la atmósfera, lo que añade energía potencial al sistema continente-océano, y a los cambios en los patrones de la temperatura superficial del mar (TSM) (Stensrud, 1996; Poveda \& Mesa, 1999). Al propiciar la actividad de convección en la atmósfera, las corrientes superficiales de chorro son importantes modeladores del clima regional; por lo tanto, el estudio de su variabilidad espaciotemporal y de los factores y fenómenos que las puedan afectar es de vital importancia para entender su influencia en la hidroclimatología de una región. El norte de Suramérica se ve afectado por dos corrientes superficiales de chorro: el chorro del Chocó (Poveda \& Mesa, 1999; Poveda, et al., 2014; Sierra, et al., 2018) y el chorro del Caribe (Amador, 2008; Wang, 2007), los cuales son mecanismos importantes en la generación de precipitación en la región (Arias, $\boldsymbol{e t}$ al., 2015; Durán-Quesada, et al., 2017).

El fenómeno de El Niño Oscilación del Sur (ENOS), en sus fases de El Niño y La Niña, también tiene un papel trascendental en la variabilidad climática de distintas regiones del planeta, entre ellas el norte de Suramérica, debido a que este fenómeno interfiere en el normal acoplamiento del sistema océano-atmósfera al inducir patrones de circulación atmosférica atípicos y de calentamiento o enfriamiento oceánico inusuales, produciendo así anomalías en los ciclos de precipitación y en los caudales de los ríos en Colombia y el norte de Suramérica (Tedeschi, et al., 2013; Poveda, et al., 2014). Las corrientes de los chorros del Chocó y del Caribe se pueden ver especialmente influenciadas por este fenómeno, ya que ambas corrientes presentan un componente geostrófico en su origen, por lo que el calentamiento del océano Pacífico durante los eventos de El Niño genera una disminución de los gradientes de temperaturas y presiones y lleva a una modificación en la intensidad de dichas corrientes (Amador, 2008; Poveda \& Mesa, 1999).

En las últimas décadas ha habido un interés creciente por estudiar los cambios que se están dando en los patrones de anomalías de la TSM en el Pacífico tropical, que se han notado desde el siglo pasado, pero que solo se empezaron a analizar y a discutir debido a la detección de un calentamiento anómalo en la región central del océano Pacífico, y enfriamientos en el este y oeste de este océano durante el verano del año 2004. En estudios posteriores se evidenció la necesidad de clasificar este calentamiento localizado del océano Pacífico como una manifestación diferente del fenómeno de El Niño Canónico, caracterizado por un calentamiento anómalo de la lengua fría localizada en el este del Pacífico tropical (Ashok, et al., 2007). Este patrón de calentamiento, más concentrado en el centro y oeste del Pacífico tropical, se ha denominado en la literatura contemporánea como El Niño Modoki (ENM) o El Niño del Pacífico Central. Algunos autores han estudiado la influencia de El Niño Modoki en la climatología de Suramérica y han establecido que este tipo de eventos de El Niño es cada vez más frecuente y puede generar efectos contrarios en la región a los observados durante los eventos de El Niño Canónico (Tedeschi, et al., 2016; Córdoba, et al., 2015).

Este trabajo se enfocó específicamente en el estudio del chorro del Chocó y del chorro del Caribe y sus variaciones durante los eventos de El Niño Canónico y El Niño Modoki. Se compararon las distintas variables atmosféricas que representan la dinámica, el transporte de humedad y la evolución temporal de estas corrientes superficiales durante dichos eventos.

\section{Datos y métodos}

Datos. Se analizaron las características climatológicas de los chorros de bajo nivel del Chocó y el Caribe y sus variaciones durante El Niño Canónico y El Niño Modoki. Dichas características se definieron mediante el análisis de vientos horizontales a $925 \mathrm{hPa}$, el corte vertical de vientos zonales en los $80^{\circ} \mathrm{W}$, el corte vertical del flujo de humedad en los $80^{\circ} \mathrm{W}$, y el ciclo anual de los vientos zonales a $925 \mathrm{hPa}$. Además, se obtuvieron los índices para determinar la intensidad de estas corrientes de chorro.

Para estos análisis se emplearon datos del reanálisis ERA-Interim del Centro Europeo de Previsiones Meteorológicas a Medio Plazo (CEPPM) (Dee, et al., 2011). Para ello se emplearon las componentes zonales y meridionales de los vientos ( $u \mathrm{y} v$, respectivamente) y la humedad específica (q) desde niveles de $1.000 \mathrm{hPa}$ hasta $700 \mathrm{hPa}$. ERAInterim cuenta con registros diarios por hora desde enero de 1979 hasta octubre de 2016, con un variado rango de resolución espacial que va desde $0,125^{\circ} \times 0,125^{\circ}$ hasta $3^{\circ} \mathrm{x}$ $3^{\circ}$. En este trabajo se usaron las series mensuales de tiempo obtenidas de los promedios diarios en una resolución espacial estándar de $0,75^{\circ} \times 0,75^{\circ}$. 
Para determinar la aparición de los eventos de El Niño Canónico y El Niño Modoki se emplearon los registros de las anomalías de la TSM de la base de datos Optimum Interpolation, Version 2 (OI V2) de la Administración Nacional Oceánica y Atmosférica del Departamento de Comercio de los Estados Unidos (National Oceanic and Atmospheric Administration, NOAA), el cual posee registros de promedios mensuales desde diciembre de 1981 hasta agosto de 2016, con una resolución espacial única de $1^{\circ} \mathrm{x}$ $1^{\circ}$ (https://www.esrl.noaa.gov/psd/data/gridded/data.noaa. oisst.v2.html).

\section{Metodología}

Los análisis desarrollados en este trabajo consideraron los cuatro trimestres del año: diciembre - enero - febrero, marzo - abril - mayo, junio - julio - agosto y septiembre - octubre - noviembre. Con la intención de enfocar los análisis en la zona de influencia de los chorros del Caribe y del Chocó, se limitó el área de estudio al norte de Suramérica. Con el objeto de caracterizar estas corrientes de chorro y determinar sus variaciones durante los eventos de El Niño Canónico y El Niño Modoki se consideraron tres características básicas: distribución horizontal de vientos a $925 \mathrm{hPa}$, cortes verticales de viento, y ciclo anual de viento zonal a $925 \mathrm{hPa}$.

Para el análisis de la distribución horizontal de vientos a $925 \mathrm{hPa}$, se consideró el dominio $110^{\circ} \mathrm{W}-50^{\circ} \mathrm{W}, 15^{\circ} \mathrm{S}-40^{\circ} \mathrm{N}$ $\mathrm{y}$, así, se calcularon los promedios multianuales de los componentes zonal y meridional del viento a $925 \mathrm{hPa}$ en los cuatro trimestres del año en cada uno de los pixeles del dominio de interés y se obtuvieron sus anomalías (con respecto al promedio de 1981 a 2016) durante los eventos de El Niño Canónico y El Niño Modoki, determinados según se describe más adelante en esta sección. Para los cortes verticales de viento y de flujo de humedad, se consideró una sección latitudinal en los $80^{\circ} \mathrm{W}$ y se obtuvo el promedio trimestral multianual del componente zonal del viento para dicha longitud, correspondiente a la costa Pacífica colombiana y el oeste del Caribe, así como para cada latitud entre $\operatorname{los} 5^{\circ} \mathrm{S}$ y $\operatorname{los} 20^{\circ} \mathrm{N}$ y para cada nivel de presión entre 1.000 hPa y $700 \mathrm{hPa}$. Este corte permite observar los núcleos de los chorros y cómo varía su posición y magnitud en cada trimestre del año. La selección de la longitud de $80^{\circ} \mathrm{W}$ radica en que en ella se pueden encontrar los dos picos de velocidades para ambos chorros (Poveda \& Mesa, 1999).

En cuanto a la determinación del ciclo anual de estas corrientes de chorro, se calcularon los promedios mensuales multianuales del viento zonal a $925 \mathrm{hPa}$ para cada latitud en el rango entre los $5^{\circ} \mathrm{S}$ y los $20^{\circ} \mathrm{N}$, lo cual permitió analizar la evolución de los chorros durante el año, pues sus núcleos se observan típicamente a este nivel de presión.

Antes de establecer la presencia de los eventos de El Niño Canónico y del Modoki, se hizo un análisis de componentes principales y funciones ortogonales empíricas (Empirical Orthogonal Functions, EOF) (Wilks, 2011) del campo de anomalías mensuales de la TSM para el dominio $20^{\circ} \mathrm{S}-20^{\circ} \mathrm{N}, 130^{\circ} \mathrm{E}-300^{\circ} \mathrm{E}$, durante el periodo de 1981 a 2016. Las EOF se obtuvieron a partir de la matriz de correlaciones.

Para determinar la presencia de los eventos del El Niño Canónico y el Modoki, se utilizaron dos índices diferentes. Para los eventos del primero se empleó el índice Niño3, que se define como el promedio de anomalías de la TSM en el área de $150^{\circ} \mathrm{W}-90^{\circ} \mathrm{W}, 5^{\circ} \mathrm{S}-5^{\circ} \mathrm{N}$. El índice Niño3.4, más comúnmente usado para determinar la presencia de dichos eventos, no se consideró, pues el área que este abarca se traslapa con las áreas de influencia del índice empleado para identificar la presencia de los eventos de El Niño Modoki, por lo que se quiso separar las regiones de mayores anomalías durante ambos tipos de evento (este del Pacífico durante eventos de El Niño Canónico y centro del Pacífico durante eventos de El Niño Modoki). Debido a que los índices previamente propuestos para determinar los eventos de El Niño Canónico no capturan el patrón anómalo de tripolo observado en la TSM del Pacífico ecuatorial durante los eventos de El Niño Modoki, Ashok, et al. (2007) han planteado un nuevo índice para describir este tipo de eventos, conocido como el índice de El Niño Modoki ( $E l$ Niño Modoki index, EMI), el cual considera las diferencias de las temperaturas en tres regiones diferentes del océano Pacífico tropical, tal que

$$
E M I=[S S T A]_{\mathrm{A}}-0.5[S S T A]_{\mathrm{B}}-0.5[S S T A]_{\mathrm{C}}
$$

donde SSTA indica las anomalías de la TSM. Las regiones están definidas de manera que la región A se encuentra entre los $165^{\circ} \mathrm{E}-140^{\circ} \mathrm{W}$ y los $10^{\circ} \mathrm{S}-10^{\circ} \mathrm{N}$, la región $\mathrm{B}$ entre los $110^{\circ}-70^{\circ} \mathrm{W}$ y los $15^{\circ} \mathrm{S}-5^{\circ} \mathrm{N}$, y la región $\mathrm{C}$ entre los $125^{\circ}-145^{\circ} \mathrm{E}$ y los $10^{\circ} \mathrm{S}-20^{\circ} \mathrm{N}$. Para identificar la presencia de eventos de El Niño Canónico y de El Niño Modoki, es necesario remover la tendencia en las series de ambos índices. Así, el umbral de decisión para clasificar eventos de estos dos tipos se fijó en 0,7, que corresponde al valor usado por Ashok, et al. (2007) y Córdoba, et al. (2014). De esta manera, fue posible identificar los dos tipos de eventos de El Niño cuando los índices respectivos eran iguales o superaban este umbral.

Al establecer todos los años en que se presentaron eventos de los dos tipos de El Niño, se analizaron las diferencias en las características generales de los chorros durante su transcurso seleccionando en cada una de las series de tiempo utilizadas para describir la climatología las mismas fechas de estos eventos. De esta manera se estableció un promedio de las variables de interés durante cada tipo de evento y se calculó la diferencia con respecto a la climatología para determinar si las anomalías en las características de los chorros presentaban cambios negativos, es decir, si disminuían, o cambios positivos, o sea, aumentaban en comparación con el promedio de largo plazo.

Una parte importante del análisis de la influencia de los eventos de El Niño Canónico y del Modoki en las dos corrientes de chorro consideradas consiste en determinar qué parte de esta variabilidad se debe precisamente a estos 
eventos y no a otros fenómenos atmosféricos. Para ello se calcularon los índices de los chorros según lo sugerido en la literatura. El índice del chorro del Caribe (Caribbean lowlevel jet-CLLJ- index) se obtuvo promediando las anomalías del viento zonal a $925 \mathrm{hPa}$ en la región $80^{\circ} \mathrm{W}-70^{\circ} \mathrm{W}$, $12,5^{\circ} \mathrm{N}-17,5^{\circ} \mathrm{N}$, y multiplicando dicho promedio por -1 , de modo que los picos de actividad de este chorro coincidieran con los valores positivos más altos del índice (Wang, 2007). Para determinar la intensidad del chorro del Chocó se consideró el promedio de las anomalías de los vientos zonales a $925 \mathrm{hPa}$ en la zona delimitada por los $80^{\circ} \mathrm{W}$ y los $5^{\circ} \mathrm{S}-7^{\circ} \mathrm{N}$ (Poveda \& Mesa, 1999). Por último, se calcularon las correlaciones entre las series de tiempo de los índices de los chorros y de los índices Niño 3 (para El Niño Canónico) y EMI (para El Niño Modoki) por trimestres y por el promedio anual. Además, se elaboraron mapas de correlaciones entre los vientos zonales a $925 \mathrm{hPa}$ y los índices Niño 3 y EMI, estableciendo la significación estadística de las correlaciones mediante la prueba $t$ de Student en un nivel del $5 \%$. Para eliminar la influencia de las tendencias en las correlaciones, antes de calcularlas se removieron las tendencias lineales de las series.

\section{Resultados y análisis}

Detección de los eventos de El Niño Canónico y El Niño Modoki. Análisis de componentes principales de la TSM. La figura 1 muestra los dos primeros modos de variabilidad de las anomalías de la TSM en el Pacífico tropical, representados por sus dos primeras EOF, los cuales explican más de la tercera parte de la variabilidad registrada en el periodo en los registros utilizados para este análisis (1981-2016). El modo de mayor aporte representó aproximadamente el 27 $\%$ de la variabilidad y en él se evidencia el patrón espacial correspondiente a un evento de El Niño Canónico (Figura 1a), con valores positivos a lo largo del ecuador desde la lengua fría del Pacífico y hacia el centro y el oeste del océano. En la evolución temporal de este modo de variabilidad se observaron distintos picos que coincidían con eventos de El Niño Canónico de gran magnitud, como los de 1998 y 2015. El segundo modo principal (Figura 1b) contribuyó con cerca del $11 \%$ de la variabilidad y representa el patrón espacial típico de los eventos de El Niño Modoki, con valores positivos en el centro del Pacífico tropical localizados hacia el oeste del Pacífico, flanqueados por valores negativos en

a)
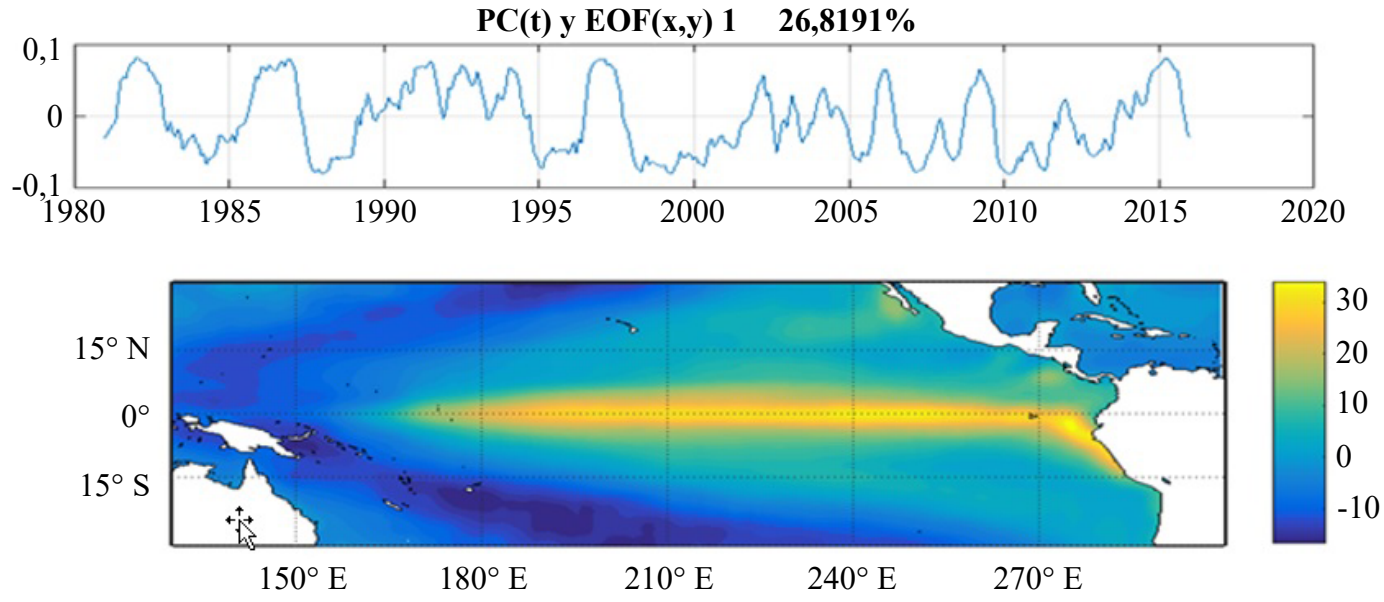

b)
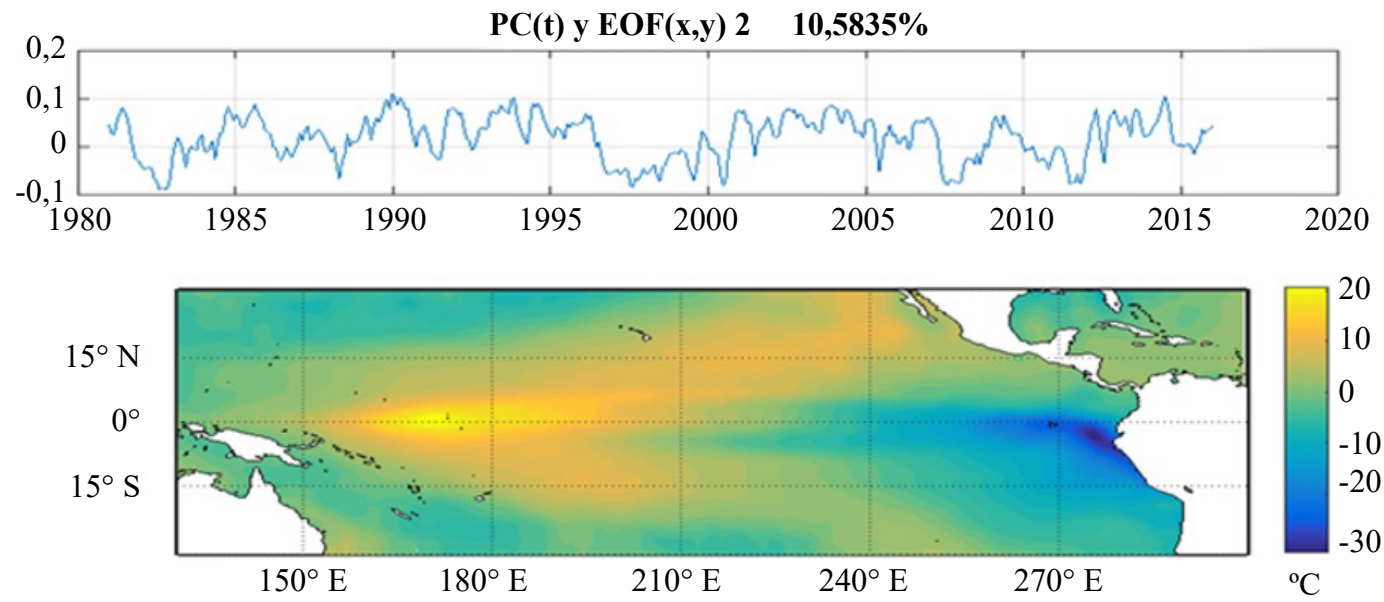

Figura 1. a) Primer modo de variabilidad de anomalías de la TSM en el Pacífico tropical (evento de El Niño Canónico). b) Segundo modo de variabilidad de anomalías de la TSM en el Pacífico tropical (evento de El Niño Modoki) 
el este, cerca de las costas de Suramérica, así como en el extremo oeste. El componente principal correspondiente a este segundo modo de variabilidad evidenció una mayor cantidad de picos en comparación con los del componente principal asociado a eventos de El Niño Canónico (Figura 1a), lo que sugiere una mayor presencia de eventos de El Niño Modoki que del Canónico durante el periodo de registro. Hubo tres modos de variabilidad que aportaron el 6, 4 y $3 \%$ del total de la varianza, respectivamente (no se muestran). Es importante aclarar que los resultados de este análisis de componentes principales pueden variar dependiendo de la base de datos que se utilice, del procesamiento previo de los datos, del procedimiento seguido para hallar los resultados y del área seleccionada para el análisis. Estos factores afectan, principalmente, el porcentaje de variabilidad explicado en cada modo, pero el patrón espacial en los dos primeros (eventos de El Niño Canónico y de El Niño Modoki, respectivamente) sigue siendo el mismo. Lo anterior se ha demostrado en otros estudios con técnicas similares a las empleadas en este trabajo, aunque los porcentajes de varianza explicada difieren entre un estudio y otro (Ashok, et al., 2007; Kao \& Yu, 2009; Deser, et al., 2010; Lian \& Chen, 2012).

Presencia de los eventos de El Niño Canónico y El Niño Modoki. La presencia de estos eventos se detectó utilizando los índices Niño3 y EMI, respectivamente, y considerando un umbral de 0,7 . Los trimestres seleccionados para cada uno de estos eventos se clasificaron según se presenta en las tablas 1 y 2 . Se seleccionaron 27 trimestres con el índice Niño 3 (eventos de El Niño Canónico), correspondientes a siete inviernos, seis primaveras, siete veranos y siete otoños boreales en el periodo de diciembre de 1981 a agosto de 2016. Los eventos de El Niño Modoki se produjeron en un número mayor de trimestres, con 41 eventos en total, correspondientes a 12 inviernos, seis primaveras, diez veranos y 14 otoños boreales. Se detectó una

Tabla 1. Trimestres en los que se presentaron eventos de El Niño Canónico

\begin{tabular}{cccc}
\hline \multicolumn{3}{c}{ Trimestres } \\
\hline Diciembre, enero, febrero & Marzo, abril, mayo & Junio, julio, agosto & Septiembre, octubre, noviembre \\
\hline $1982 / 83$ & 1983 & 1982 & 1982 \\
$1986 / 87$ & 1987 & 1983 & 1987 \\
$1991 / 92$ & 1992 & 1987 & 1997 \\
$1997 / 98$ & 1993 & 1991 & 2002 \\
$2006 / 7$ & 1998 & 1997 & 2006 \\
$2009 / 10$ & 2016 & 2009 & 2009 \\
$2015 / 16$ & & 2015 & 2015 \\
\hline
\end{tabular}

Tabla 2. Trimestres en los que se presentaron eventos de El Niño Modoki

\begin{tabular}{cccc}
\hline & TRIMESTRES ENM & \\
\hline Diciembre, enero, febrero & Marzo, abril, mayo & Junio, julio, agosto & Septiembre, octubre, noviembre \\
\hline $1986 / 87$ & 1991 & 1982 & 1982 \\
$1990 / 91$ & 1993 & 1987 & 1986 \\
$1991 / 92$ & 1995 & 1990 & 1987 \\
$1992 / 93$ & 2007 & 1991 & 1990 \\
$1993 / 94$ & 2015 & 1992 & 1991 \\
$1994 / 95$ & 2016 & 1993 & 1993 \\
$2001 / 2002$ & & 1994 & 1994 \\
$2002 / 2003$ & & 2002 & 2002 \\
$2004 / 2005$ & & 2004 & 2004 \\
$2009 / 2010$ & & 2015 & 2006 \\
$2014 / 2015$ & & & 2009 \\
$2015 / 2016$ & & & 2012 \\
& & & 2014 \\
\end{tabular}


mayor presencia de eventos de El Niño Modoki durante las estaciones de otoño e invierno boreales. En general, estos eventos sucedieron con mayor frecuencia en el Pacífico tropical durante el periodo de análisis. En comparación con otros estudios en los que también se determinó la presencia de estos dos eventos (Tedeschi, et al., 2013; Córdoba, et al., 2014), se encontró una coincidencia casi total en cuanto a los trimestres seleccionados, con algunas diferencias atribuibles al uso de distintas bases de datos.

En la figura 1S, https://www.raccefyn.co/index.php/ raccefyn/article/downloadSuppFile/705/3142 se presentan las series de tiempo de los índices Niño3 y EMI, indicando el umbral de 0,7 para establecer la presencia de eventos de El Niño Canónico y del Modoki, respectivamente. En la serie de tiempo del índice Niño3, se observan tres picos de magnitud importante que están relacionados con la fase positiva del ENOS, los cuales ocurrieron en los años de 1983, 1998 y 2015, siendo el evento de El Niño Canónico de 1998 el de mayor magnitud durante el periodo de 1981 a 2016. En la serie de tiempo en la que se aplicó el índice EMI, no se presentaron picos de una magnitud comparable a los del Niño3, pero sí se cruzó el umbral en más ocasiones, lo que indica una mayor presencia de este tipo de eventos. Los eventos de este tipo con mayor intensidad ocurrieron durante la fase negativa, es decir, La Niña Modoki. Algunos eventos de El Niño Modoki de magnitud considerable se produjeron en los años de 1992, 1995, 2004 y 2010.

Climatología de las corrientes superficiales de chorro. Vientos a $925 \mathrm{hPa}$. La figura 2 presenta la climatología trimestral de vientos horizontales a $925 \mathrm{hPa}$ en el norte de Suramérica durante el periodo comprendido entre enero de 1979 y agosto de 2016. La distribución espacial de los vientos asociados al CLLJ evidenció un ciclo anual con una primera temporada de fortalecimiento durante el trimestre de diciembre-enero-febrero y velocidades entre $14 \mathrm{~m} / \mathrm{s}$ y 16 $\mathrm{m} / \mathrm{s}$ (Figura 2a); durante marzo-abril-mayo se presentaba
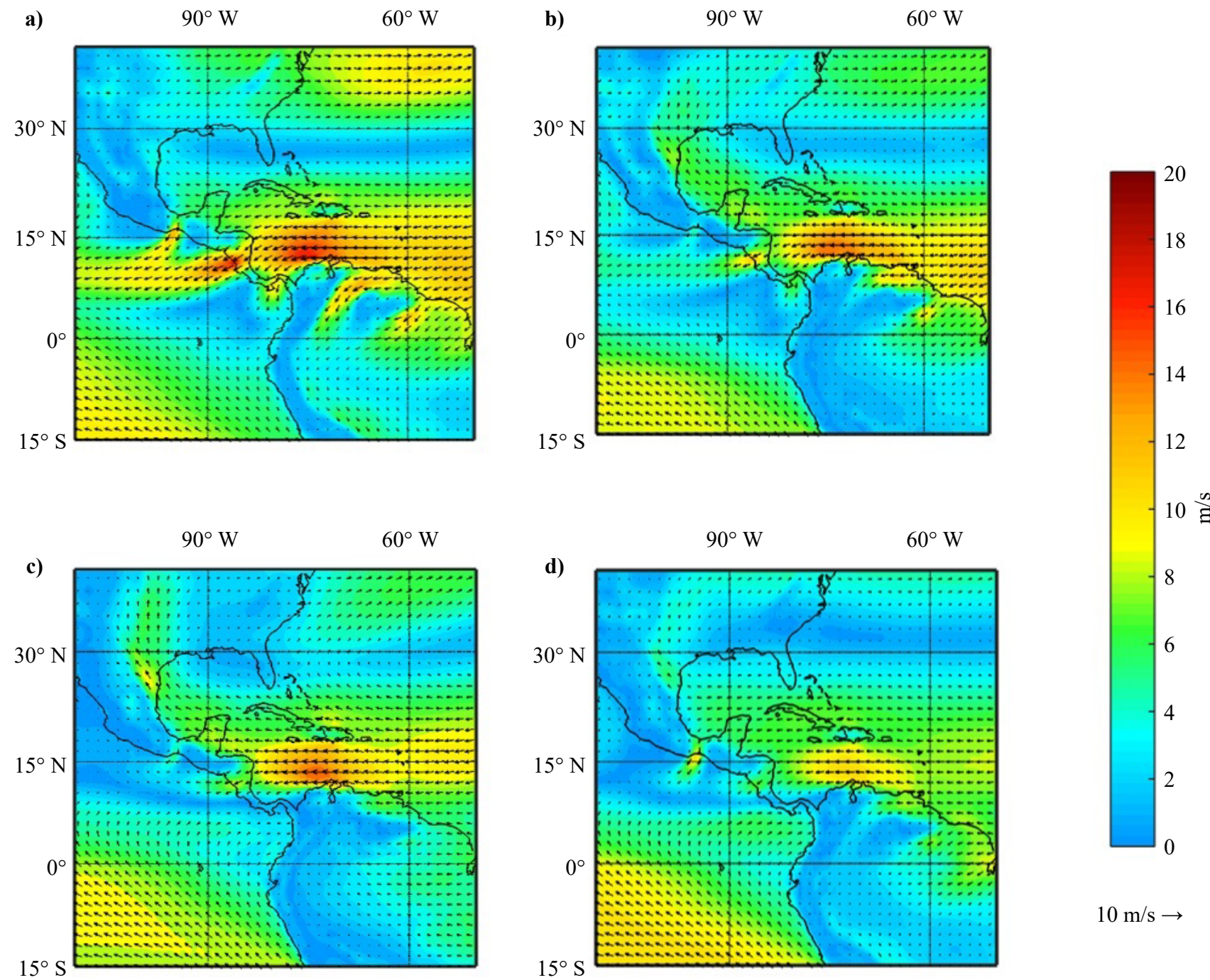

Figura 2. Climatología de los vientos a $925 \mathrm{hPa}$ durante el periodo 1979 - 2016 para los trimestres de a) diciembre-enero-febrero, b) marzoabril-mayo, c) junio-julio-agosto y d) septiembre-octubre-noviembre 
un debilitamiento de estos vientos (Figura $2 b$ ), que volvían a fortalecerse durante junio-julio-agosto, alcanzando velocidades entre $12 \mathrm{~m} / \mathrm{s}$ y $14 \mathrm{~m} / \mathrm{s}$ (Figura 2c), y, por último, las menores intensidades del CLLJ se observaron en septiembre-octubre-noviembre, con velocidades de $8 \mathrm{~m} / \mathrm{s}$ a $10 \mathrm{~m} / \mathrm{s}$ (Figura 2d). Además, durante la temporada der junio-julio-agosto se observó una bifurcación del CLLJ, al conformarse la corriente de chorro de las Grandes Planicies (Great Plains low-level jet, GPLLJ) (Cook \& Vizy, 2010).

Para el chorro del Chocó, solo se detectó un pico anual en las velocidades, el cual alcanzó valores entre $5 \mathrm{~m} / \mathrm{s}$ y 7 $\mathrm{m} / \mathrm{s}$ durante el trimestre de septiembre-octubre-noviembre; las velocidades más bajas se observaron durante los trimestres de diciembre-enero-febrero (Figura 2a) y marzoabril-mayo (Figura 2b), con magnitudes no superiores a 3 $\mathrm{m} / \mathrm{s}$. En general, las velocidades en el CLLJ fueron mayores que en el chorro del Chocó en todas las estaciones del año. Además, en el CLLJ se evidenció un claro ciclo bimodal, en tanto que en el chorro del Chocó fue unimodal.

Corte vertical de viento zonal en los $80^{\circ} \mathrm{W}$. La figura 3 muestra un corte vertical de vientos zonales en los $80^{\circ} \mathrm{W}$ para cada trimestre del año. Alrededor de $\operatorname{los} 15^{\circ} \mathrm{N}$, con vientos entre los 950 y $700 \mathrm{hPa}$, se puede ver el perfil del CLLJ, caracterizado por valores negativos de velocidad zonal durante todo el año. Por su parte, el perfil del chorro del
Chocó se distingue cerca de los $5^{\circ} \mathrm{N}$, con vientos entre 1.000 y $900 \mathrm{hPa}$, caracterizado por valores positivos de velocidad zonal, principalmente para el trimestre de septiembre a noviembre. En la región que representa el núcleo del CLLJ se pueden observar los dos picos de velocidades descritos con los vientos a $925 \mathrm{hPa}$ durante los trimestres de diciembre-enero-febrero (Figura 3a) y junio-julio-agosto (Figura 3c), siendo de mayor magnitud durante este último, lo que produjo que dicho núcleo se expandiera a capas más altas de la atmósfera en este trimestre que en los otros, en los cuales no pasó de los $800 \mathrm{hPa}$. El núcleo del CLLJ estuvo presente a lo largo del año alrededor de $\operatorname{los} 15^{\circ} \mathrm{N}$.

En el extremo sur se puede reconocer el perfil que representa el chorro del Chocó, con su respectivo ciclo anual con un solo pico de velocidades de vientos zonales durante el trimestre de septiembre-octubre-noviembre. A medida que este chorro se fortalece, su núcleo se desplaza hacia el norte y a la vez se expande latitudinalmente, abarcando regiones que van aproximadamente desde los $5^{\circ} \mathrm{S}$ hasta los $10^{\circ} \mathrm{N}$ durante el trimestre de mayores velocidades. Por el contrario, durante el trimestre de menores velocidades (marzo-abrilmayo), este chorro solo llega a abarcar latitudes desde los $5^{\circ} \mathrm{S}$ hasta $\operatorname{los} 5^{\circ} \mathrm{N}$. En cuanto a la altura del chorro del Chocó, en ningún momento del año alcanza a influenciar las capas por encima de los $800 \mathrm{hPa}$. a)

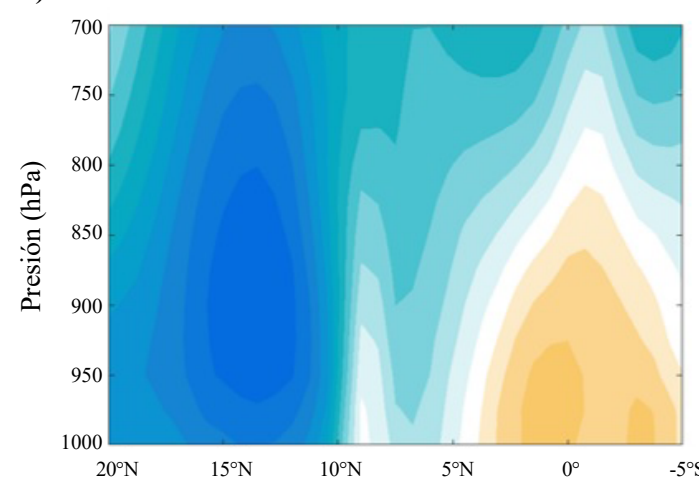

c)

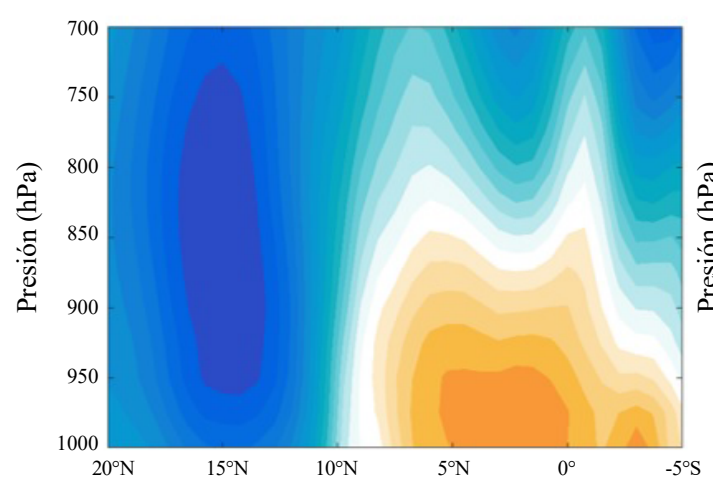

b)

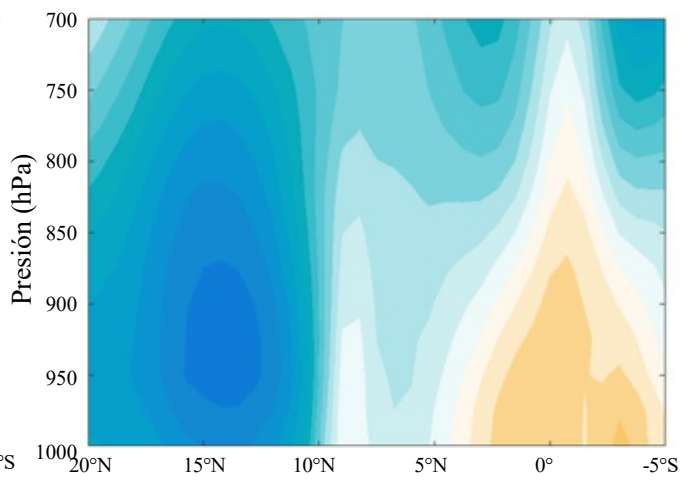

d)

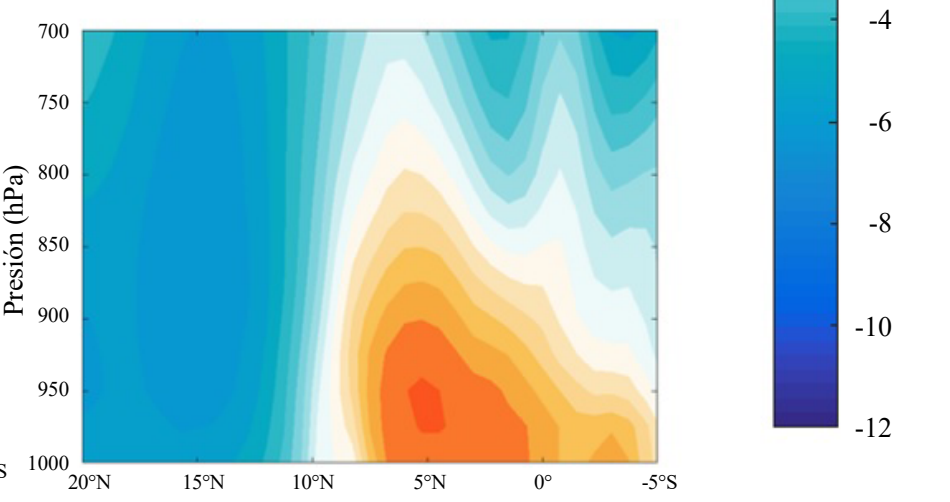

Figura 3. Climatología de corte vertical de los vientos zonales en los $80^{\circ} \mathrm{W}$ durante el periodo 1979 - 2016 para los trimestres de a) diciembre-enero-febrero, b) marzo-abril-mayo, c) junio-julio-agosto y d) septiembre-octubre-noviembre 
Por otra parte, un análisis del transporte de humedad integrado en la vertical (no se muestra), indicó que el flujo de humedad asociado con estas corrientes fue directamente proporcional a la velocidad del viento zonal, aunque los núcleos de mayor transporte estuvieron más cerca de la superficie.

Ciclo anual de viento zonal en los $80^{\circ} \mathrm{W}$ y a $925 \mathrm{hPa}$. En la figura 4 se presenta el ciclo anual de los vientos zonales en los $80^{\circ} \mathrm{W}$ y a $925 \mathrm{hPa}$. Se puede observar, desde otra perspectiva, la evolución de la velocidad y la posición de los chorros a lo largo del año. En esta visualización se aprecian, de una forma más detallada, los cambios en la magnitud y la posición del núcleo de cada uno de los chorros. En este ciclo anual se comprobó cómo el CLLJ desarrolló dos fases de velocidades máximas a lo largo del año: específicamente

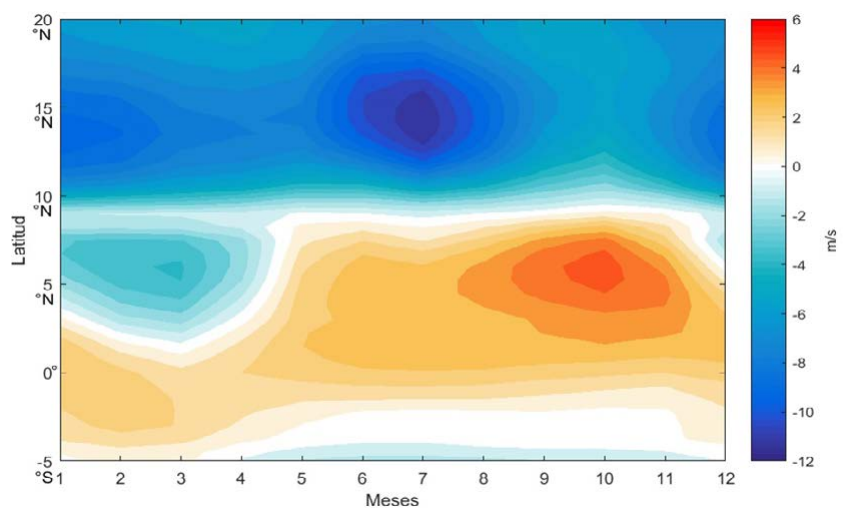

Figura 4. Climatología del ciclo anual de los vientos zonales a 925 $\mathrm{hPa}$ en una longitud de $80^{\circ} \mathrm{W}$ para el periodo $1979-2016$ durante el mes de julio se observaron velocidades superiores a $\operatorname{los} 10 \mathrm{~m} / \mathrm{s}$, en tanto que se observó un pico menor en enero. El núcleo del CLLJ no sufrió cambios notables en su posición: durante la época de mayor velocidad (julio) se posicionó en $\operatorname{los} 15^{\circ} \mathrm{N}$, en tanto que en enero estuvo un poco más al sur, alrededor de los $14^{\circ} \mathrm{N}$. Con el chorro del Chocó sucedió también lo descrito para los perfiles verticales y la distribución horizontal de los vientos a $925 \mathrm{hPa}$, con único pico de velocidad específicamente en el mes de octubre y una posición desplazada hacia el norte que abarcó un rango de latitudes más amplio al exhibir mayor intensidad.

Cambios en las corrientes de chorro durante los eventos de El Niño Canónico y El Niño Modoki. Vientos a $925 \mathrm{hPa}$. La figura 5 muestra las anomalías del viento zonal a $925 \mathrm{hPa}$ en el norte de Suramérica y el Caribe en los cuatro trimestres del año durante los eventos de El Niño Canónico (Figuras 5a - 5d) y El Niño Modoki (Figuras 5e - 5h). En general, las anomalías en las velocidades del CLLJ fueron mayores durante los eventos de El Niño Canónico. En cuanto al chorro del Chocó, también se observó una mayor variación en sus anomalías durante los eventos de este tipo. El trimestre de diciembre-enero-febrero, en particular, se caracterizó por un fortalecimiento de esta corriente durante ambos eventos de El Niño, en tanto que en el trimestre de marzo-abril-mayo su velocidad disminuyó durante los eventos de El Niño Modoki, pero se fortaleció levemente en los eventos de El Niño Canónico. Durante los trimestres de junio-julio-agosto y septiembre-octubre-noviembre se observó un debilitamiento de esta corriente durante ambos tipos de eventos, siendo mayores las anomalías asociadas con El Niño Canónico.
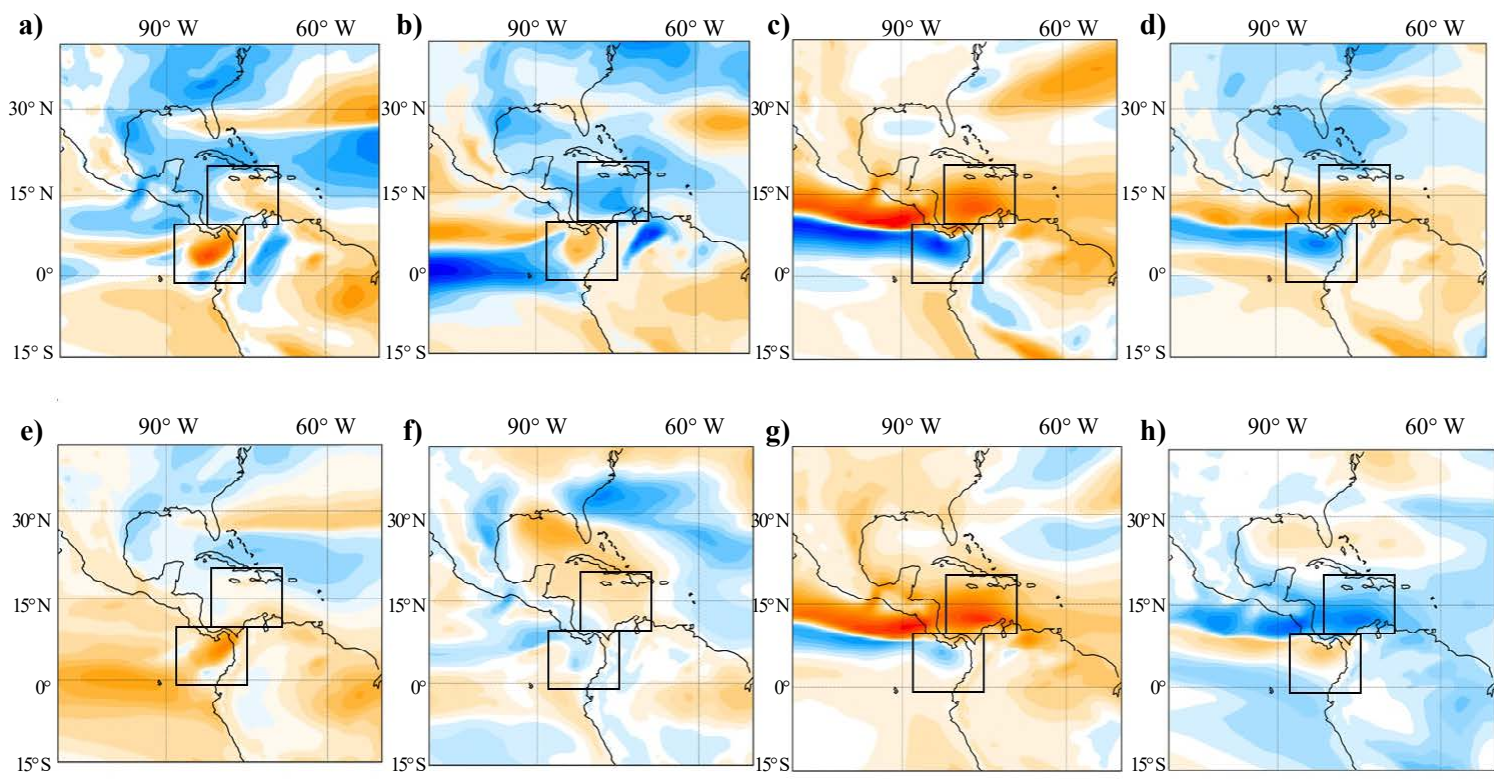

Figura 5. Anomalías de vientos horizontales a $925 \mathrm{hPa}$ durante los eventos de El Niño Canónico en los trimestres de: a) diciembreenero-febrero, b) marzo-abril-mayo, c) junio-julio-agosto y d) septiembre-octubre-noviembre. Anomalías de vientos horizontales a 925 hPa durante los eventos de El Niño Modoki en los trimestres de: e) diciembre-enero-febrero, f) marzo-abril-mayo, g) junio-julio-agosto y h) septiembre-octubre-noviembre. Los recuadros muestran las regiones de aparición de los chorros del Caribe (norte) y el Chocó (sur). 
Corte vertical del viento zonal en los $80^{\circ} \mathrm{W}$. La figura 6 muestra las anomalías en los perfiles verticales del viento zonal en los $80^{\circ} \mathrm{W}$ durante los eventos de El Niño Canónico (Figuras $6^{\mathrm{a}}$ - 6d) y El Niño Modoki (Figuras 6e - 6f). Las anomalías negativas observadas en la región de influencia del CLLJ (alrededor de los $15^{\circ} \mathrm{N}$ ) indican un fortalecimiento de esta corriente, mientras que los valores positivos en esta región sugieren un debilitamiento. En el chorro del Chocó (que ocurre en latitudes más al sur, entre los $5^{\circ} \mathrm{S}$ y $\operatorname{los} 7^{\circ} \mathrm{N}$ ), las anomalías positivas indican un fortalecimiento de este chorro mientras que las negativas indican su debilitamiento. Durante diciembre-enero-febrero (Figura 6a), el componente zonal del CLLJ se debilitó en los eventos de El Niño Canónico; por su parte, durante los eventos de El Niño Modoki se observó un debilitamiento más leve de esta corriente, con algunos sectores de fortalecimiento hacia el norte y de debilitamiento hacia el sur.

En general, se identificó que el chorro del Chocó se debilitó en diciembre-enero-febrero y en marzo-abril-mayo durante El Niño Canónico, en tanto que durante El Niño Modoki se observaron diferencias de pequeña magnitud, con un leve debilitamiento alrededor de $\operatorname{los} 5^{\circ} \mathrm{N}$ en diciembreenero-febrero y un fortalecimiento en marzo-abril-mayo. En la segunda mitad del año (junio-julio-agosto y septiembreoctubre-noviembre), se observó un debilitamiento del chorro del Chocó durante los dos tipos de eventos de El Niño, siendo de mayor magnitud el impacto del Canónico. Estos resultados coinciden con lo observado en la distribución espacial de los vientos durante ambos tipos de eventos El Niño (Figura 5).

Ciclo anual del viento zonal en los $80^{\circ} \mathrm{W}$ y a $925 \mathrm{hPa}$. La figura $2 \mathrm{~S}$, https://www.raccefyn.co/index.php/raccefyn/ article/downloadSuppFile/705/3143, muestra el ciclo anual de anomalías de la velocidad del viento zonal en los $80^{\circ} \mathrm{W}$ y a $925 \mathrm{hPa}$ durante los eventos de El Niño Canónico (Figura 2Sa) y El Niño Modoki (Figura 2Sb). Para los eventos de El Niño Canónico se observó que el CLLJ se debilitaba entre enero y mayo, en tanto que desde junio hasta diciembre se fortalecía. Por su parte, el chorro del Chocó se debilitaba a lo largo de los 12 meses del año durante los eventos de El Niño Canónico. Debido a que estas figuras solo muestran el nivel de los $925 \mathrm{hPa}$, que es donde se encuentra el núcleo de ambos chorros, no se observan los debilitamientos en las capas superiores del chorro del Chocó identificados en su estructura vertical (Figura 3).

En los eventos El Niño Modoki se observó un debilitamiento del CLLJ en enero y febrero; el resto del año, este chorro se fortalecía, presentando los mayores cambios durante junio y agosto. En contraste, el núcleo del chorro del Chocó se debilitaba en los dos primeros meses del año, se fortalecía entre marzo y abril, y se debilitaba nuevamente en el resto del año.

Análisis de correlaciones. Para profundizar en la posible relación entre los dos tipos de eventos de El Niño y las circulaciones del chorro en estudio, se elaboraron mapas de correlación entre la componente zonal del viento a $925 \mathrm{hPa}$ y los índices EMI (Figuras 7a - 7d) y Niño 3 (Figuras 7e - 7h). La significación estadística de la correlación se determinó mediante una prueba $t$ de Student de dos colas con un nivel de confianza de $95 \%$. Así, cuando los coeficientes de correlación no superaron el umbral indicado por la prueba, se consideró que no representaban adecuadamente la relación entre ambas variables y se les asignó un valor de 0 .

Los resultados obtenidos indican que ambos tipos de eventos de El Niño se asociaron con un fortalecimiento del CLLJ durante diciembre-enero-febrero, y con un debilitamiento durante junio-julio-agosto y septiembre-octubrenoviembre, principalmente durante El Niño Canónico. Esto

a)

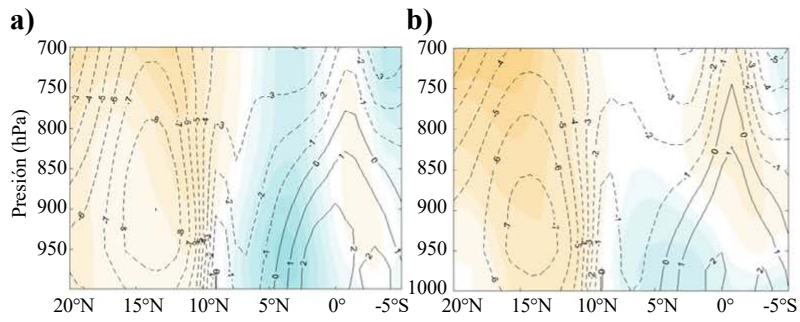

c)

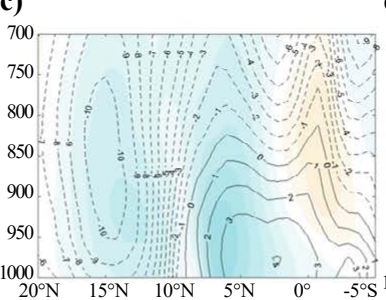

e)

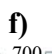

g)
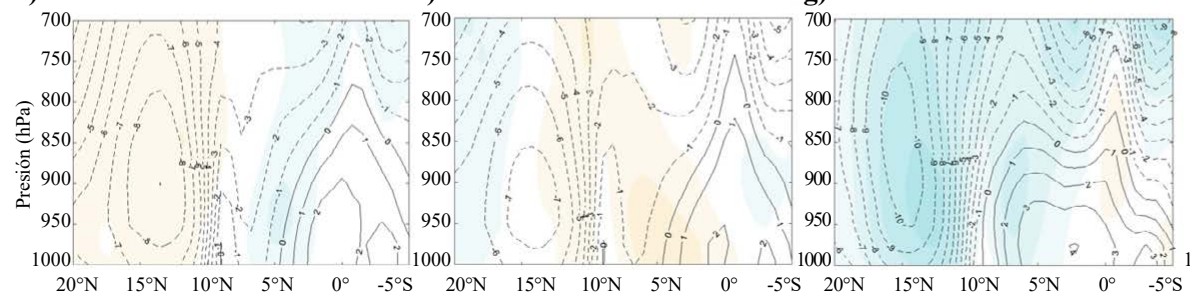

d)

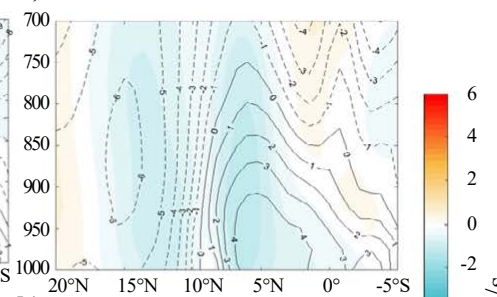

h)

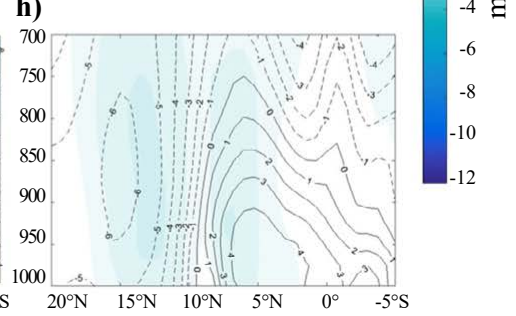

Figura 6. Anomalías de corte vertical de los vientos zonales en los $80^{\circ} \mathrm{W}$ durante los eventos de El Niño Canónico en los trimestres de: a) diciembre-enero-febrero, b) marzo-abril-mayo, c) junio-julio-agosto y d) septiembre-octubre-noviembre. Anomalías de corte vertical de los vientos zonales en los $80^{\circ} \mathrm{W}$ durante los eventos de El Niño Modoki en los trimestres de: e) diciembre-enero-febrero, f) marzo-abril-mayo, g) junio-julio-agosto y h) septiembre-octubre-noviembre. La climatología se presenta en contornos. 
a)

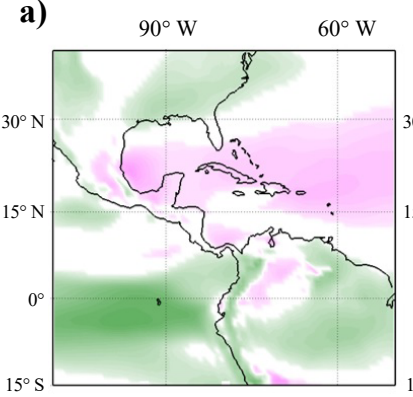

e)

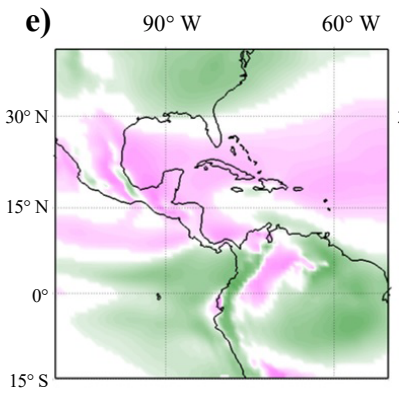

b)

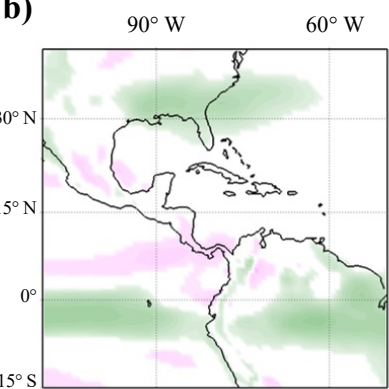

c)

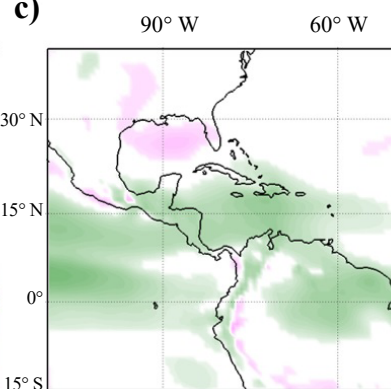

d)
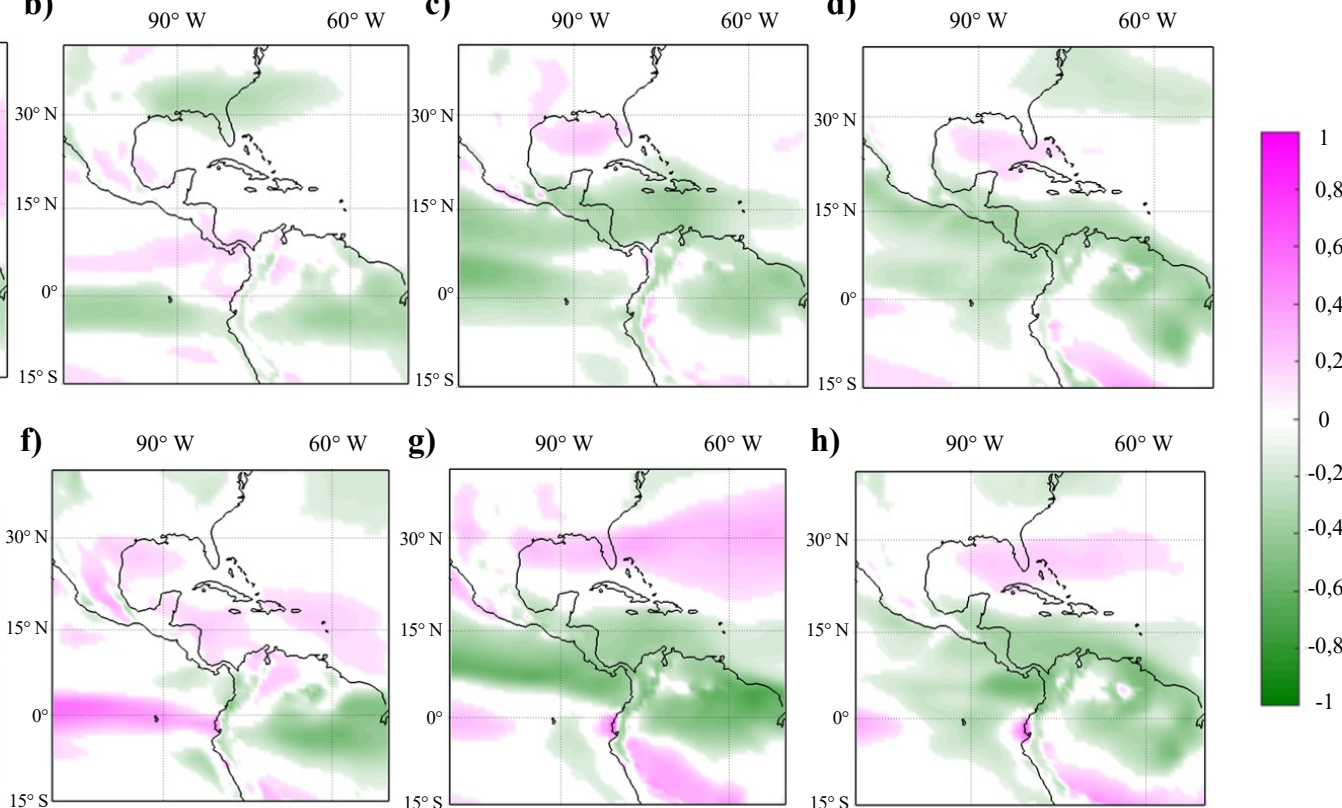

g)

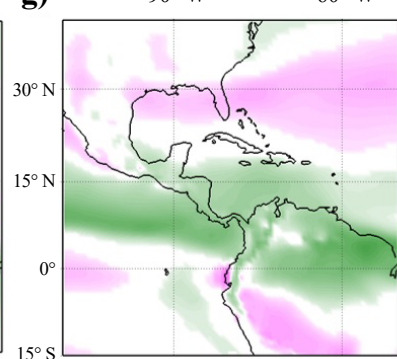

h)

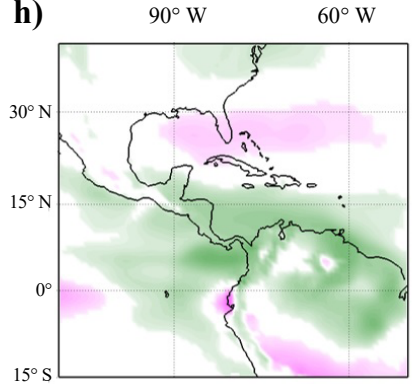

0

$-0,2$

$-0,4$

$-0,6$ $-0,8$

Figura 7. Mapas de correlación entre el componente zonal del viento a $925 \mathrm{hPa}$ y el índice EMI en los trimestres de: a) diciembre-enerofebrero, b) marzo-abril-mayo, c) junio-julio-agosto y d) septiembre-octubre-noviembre. Mapas de correlación entre el componente zonal del viento a $925 \mathrm{hPa}$ y el índice Niño 3 en los trimestres de: e) diciembre-enero-febrero, f) marzo-abril-mayo, g) junio-julio-agosto y h) septiembre-octubre-noviembre. Las correlaciones presentadas en los mapas fueron estadísticamente significativas según una prueba t de Student con un nivel de significación del $5 \%$.

coincide con lo reportado en estudios anteriores sobre cómo los campos de la TSM y la presión a nivel del mar presentan un comportamiento opuesto durante el invierno y el verano boreal en la fase positiva del fenómeno ENOS (Wang, 2007). Por su parte, el chorro del Chocó se ve debilitado durante estos eventos, excepto en el trimestre de marzoabril-mayo, cuando se observa su fortalecimiento durante los eventos de El Niño Modoki. Estos resultados concuerdan con lo observado en la distribución espacial y vertical de estas corrientes (Figuras 5 y 6 ).

En general, nuestros resultados identificaron que los eventos de El Niño Canónico y El Niño Modoki generaron impactos similares en las corrientes de chorro analizadas durante la mayoría de los trimestres, exceptuando el de marzo-abril-mayo, en el que se observaron impactos opuestos de los dos tipos de eventos. Sin embargo, durante los eventos de El Niño canónico se presentaron impactos de mayor magnitud en estas corrientes que durante los eventos de El Niño Modoki. El mayor impacto en estas corrientes de chorro durante El Niño Canónico puede radicar en que durante estos eventos los gradientes de temperatura y presión entre el este del Pacífico tropical y el occidente colombiano se reducen más en comparación con los observados durante El Niño Modoki, debido a un calentamiento más pronunciado en el este del Pacífico durante El Niño Canónico. Este gradiente es particularmente importante en la dinámica del chorro del Chocó (Poveda \& Mesa, 1999; Sierra, et al., 2018).
Lo anterior es de particular importancia en el pronóstico hidrometeorológico en Colombia, pues el tipo de calentamiento que se genera en el océano Pacífico tropical, como es el típico durante los eventos de El Niño Canónico (concentrado en el este) o durante los de El Niño Modoki (concentrado en el centro), influye en la intensidad que pueden tener las corrientes de chorro del Chocó y el Caribe, y a su vez, el transporte de humedad atmosférica desde los océanos Pacífico y Atlántico asociados con estas corrientes.

\section{Conclusiones}

En estudios anteriores se ha determinado que las corrientes de chorro de bajo nivel del Chocó y el Caribe son mecanismos importantes para el transporte de humedad y la generación de precipitación en el norte de Suramérica, y contribuyen a la advección de vapor de agua y calor hacia esta región, lo que los convierte en importantes modeladores del clima local (Poveda \& Mesa, 1999; Arias, $\boldsymbol{e t}$ al., 2015; Hoyos, et al., 2018). El chorro del Chocó sigue una trayectoria paralela a la costa oeste de Suramérica y al cruzar el ecuador se recurva, penetrando en las costas colombianas. Por su parte, el CLLJ circula desde el océano Atlántico tropical y llega al mar Caribe donde se divide en dos ramas en el verano boreal, una que cruza el Golfo de Yucatán y se une al GPLLJ en Estados Unidos, y otra que cruza Centroamérica y se une al chorro del Chocó. Cada uno de estos chorros posee diferentes regímenes de velocidad. El chorro del Chocó posee un ciclo anual con 
picos en el trimestre de septiembre-octubre-noviembre, con un núcleo de velocidades que se desplaza hacia el norte a medida y se fortalece durante el verano y el otoño boreales. Por su parte, el CLLJ tiene un ciclo semianual, con picos en diciembre-enero-febrero y junio-julio-agosto, y su núcleo está ubicado a $\operatorname{los} 15^{\circ} \mathrm{N}$ sin presentar migraciones latitudinales significativas durante el año, como lo hace el chorro del Chocó.

En diversos estudios se ha establecido la relevancia del fenómeno de ENOS en la variabilidad interanual de la hidroclimatología del norte de Suramérica y el Caribe. Específicamente, se ha determinado que los eventos de El Niño se asocian a la reducción de la precipitación y los caudales en Colombia, en tanto que La Niña genera lo opuesto. Cada vez hay más investigación sobre los efectos que tienen las diversas formas de calentamiento o enfriamiento del Pacífico tropical asociadas con el ENOS. En este sentido, se ha encontrado que el calentamiento del océano Pacífico tropical observado durante los eventos de El Niño no siempre se localiza en la región este del océano (lo que se ha denominado El Niño Canónico), sino que, en algunos eventos, puede presentarse concentrado en el centro de este océano y con menor intensidad (lo que se ha denominado El Niño Modoki). Nuestro análisis de componentes principales permitió establecer los dos principales modos de variabilidad del campo de anomalías de la TSM en el Pacífico tropical, y evidenció que su primer modo de variabilidad se asocia con eventos de El Niño Canónico (caracterizados por un calentamiento pronunciado en el este del Pacífico tropical), lo que explicó el 26,8 \% de la variabilidad de este campo espacial, en tanto que el segundo modo se asoció a eventos de El Niño Modoki, los cuales dieron cuenta de $10,58 \%$ de la varianza. Además, se pudo determinar que estos últimos fueron más frecuentes que los de El Niño Canónico durante el periodo considerado en este estudio (19812016), presentándose principalmente en los trimestres de diciembre-enero-febrero y septiembre-octubre-noviembre, es decir, el invierno y el otoño boreales, respectivamente.

Como forma de determinar los posibles impactos de estos dos eventos en el clima de Colombia, en este estudio se profundizó en su influencia sobre las corrientes de chorro del Chocó y el Caribe. Los resultados sugieren que los dos eventos generaron impactos similares en las corrientes de chorro analizadas durante la mayoría de los trimestres. En este sentido, ambos tipos de eventos se asociaron con un fortalecimiento del CLLJ durante diciembre-enero-febrero y marzo-abril-mayo, y con un debilitamiento durante juniojulio-agosto y septiembre-octubre-noviembre, principalmente durante El Niño Canónico. Por su parte, el chorro del Chocó se vio debilitado durante estos eventos, excepto en el trimestre de marzo-abril-mayo, cuando se observó su fortalecimiento durante los eventos de El Niño Modoki.

En otros estudios se ha señalado que las anomalías en estas corrientes de chorro pueden estar relacionadas con anomalías en la precipitación en Colombia. Un ejemplo de ello lo constituye lo ocurrido en el país entre 2010 y 2012, cuando un evento de La Niña de gran magnitud generó dos de las temporadas lluviosas más fuertes de los últimos años (Hoyos, et al., 2013). El enfriamiento generalizado del océano Pacífico tropical, sumado al calentamiento inusual del mar Caribe ocurrido durante este período, generó un fortalecimiento del chorro del Chocó y un debilitamiento del CLLJ, lo que permitió el transporte de mayores cantidades de vapor de agua hacia Colombia y el incremento generalizado de precipitación en todo el país (Arias, et al., 2015). Esto concuerda con los resultados obtenidos en esta investigación: durante los eventos de El Niño canónico se observó un debilitamiento del chorro del Chocó, principalmente en junio-julio-agosto y septiembre-octubrenoviembre, lo que se asoció con un menor transporte de humedad desde el océano Pacífico (Arias, et al., 2015), contribuyendo a la reducción de la precipitación observada durante estos eventos en Colombia.

Esta investigación aporta nuevos elementos sobre la influencia del fenómeno ENOS en Colombia, profundizando en la importancia que tiene el patrón de calentamiento o enfriamiento del Pacífico tropical en la variabilidad interanual de la hidroclimatología del país. Nuestro aporte radica, especialmente, en que se pudo establecer que el comportamiento de las corrientes de chorro del Caribe y el Chocó, factores importantes en la determinación de los patrones de precipitación en Colombia, se ven modificados según la presencia de los eventos de El Niño Canónico y El Niño Modoki. Esto es fundamental en lo que a pronóstico de precipitación y caudales se refiere, pues la respuesta de la precipitación (y de los mecanismos asociados con ella, como las corrientes de chorro) exhibe una mayor variación durante los eventos canónicos de El Niño, en tanto que los eventos Modoki ejercen una influencia más atenuada. Lo anterior es importante para generar estrategias de adaptación y mitigación frente a las temporadas lluviosas o secas anómalas que se puedan presentar durante dichos eventos.

\section{Información suplementaria}

Figura 1S. Series de tiempo de los índices Niño3 y EMI durante el periodo 1980-2016. Vea la figura 1S en: https://www.raccefyn.co/ index.php/raccefyn/article/downloadSuppFile/705/3142

Figura 2S. Ciclo anual de anomalías (colores) y climatología (contornos) de los vientos zonales en $\operatorname{los} 80^{\circ} \mathrm{W}$ y a $925 \mathrm{hPa}$ durante los eventos de: a) El Niño Canónico y b) El Niño Modoki. Vea la figura $2 \mathrm{~S}$ en: https://www.raccefyn.co/index.php/raccefyn/article/ downloadSuppFile/705/3143

\section{Agradecimientos}

Este trabajo fue financiado por la convocatoria CODI para Trabajos de Grado 2016/1 de la Universidad de Antioquia. 


\section{Contribución de los autores}

Lina M. Serna procesó la información y realizó los análisis aquí presentados; Paola A. Arias y Sara C. Vieira diseñaron y dirigieron la investigación.

\section{Conflicto de intereses}

Los autores manifiestan no presentar conflicto de intereses para este trabajo.

\section{Referencias}

Amador, J. (2008). The Intra-Americas Sea Low-level Jet Overview and Future Research. Trends and Directions in Climate Research: Ann. N.Y. Acad. Sci. 1146: 153-188.

Arias, P. A, Martínez, J. A., Vieira, S. C. (2015). Moisture sources to the 2010-2012 anomalous wet season in northern South America. Climate Dynamics. 45: 2861-2884.

Ashok, K., Behera, S. K., Rao, S. A., Weng, H., Yamagata, T. (2007). El Niño Modoki and its possible teleconnection, J. Geophys. Res. 112: 1-27. Disponible en: https://agupubs. onlinelibrary.wiley.com/doi/epdf/10.1029/2006JC003798

Cook, K. H. \& Vizy, E. K. (2010). Hydrodynamics of the Caribbean Low-Level Jet and Its Relationship to Precipitation. J. Climate. 23: 1477-1494.

Córdoba, S., Palomino, R., Gámiz, S., Castro, Y., Esteban, R. (2014). Assessing the impact of El Niño Modoki on seasonal precipitation in Colombia. Global and Planetary Change. 124: 41-61.

Córdoba, S., Palomino, R., Gámiz, S., Castro, Y., Esteban, R. (2015). Influence of tropical Pacific TSM on seasonal precipitation in Colombia: Prediction using El Niño and El Niño Modoki. Clim Dyn. 44: 1293-1310.

Dee, D. P., Uppala, S. M., Simmons, A. J., Berrisford, P., Poli, P., Kobayashi, S., Andrae, U., Balmaseda, M. A., Balsamo, G., Bauer, P., Bechtold, P., Beljaars, A. C. M., van de Berg, L., Bidlot, J., Bormann, N., Delsol, C., Dragani, R., Fuentes, M., Geer, A. J., Haimberger, L., Healy, S. B., Hersbach, H., Hólm, E. V., Isaksen, L., Kållberg, P., Köhler, M., Matricardi, M., McNally, A. P., MongeSanz, B. M., Morcrette, J.-J., Park, B.-K., Peubey, C., de Rosnay, P., Tavolato, C., Thépaut, J.N., Vitart, F. (2011). The ERA-Interim reanalysis: Configuration and performance of the data assimilation system. Q.J.R. Meteorol. Soc. 137: 553-597.

Deser, C., Alexander, M., Xie, S., Phillips, A. (2010). Sea Surface Temperature Variability: Patterns and Mechanisms. Annu. Rev. Mar. Sci. 2: 115-143.

Durán Quesada, A. M., Gimeno, L., Amador, J. (2017). Role of moisture transport for Central American precipitation. Earth System Dynamics. 8: 147-161.
Hoyos, I., F. Dominguez, J. Cañón-Barriga, J.A. Martínez, R. Nieto, L. Gimeno, and P. A. Dirmeyer (2018). Moisture origin and transport processes in Colombia, northern South America. Clim. Dyn. 50 (3-4): 971-990. Disponible en: https://link.springer.com/article/10.1007/s00382-0173653-6

Hoyos, N., Escobar, J., Restrepo, J. C., Arango, A. M., Ortiz, J. C. (2013). Impact of the 2010-2011 La Niña phenomenon in Colombia, South America: The human toll of an extreme weather event. Applied Geography. 39: 16-25.

Kao, H. \& Yu, J. (2009). Contrasting Eastern-Pacific and CentralPacific Types of ENSO. J. Climate. 22: 615-632.

Lian, T. \& Chen, D. (2012). An Evaluation of Rotated EOF Analysis and Its Application to Tropical Pacific TSM Variability. J. Climate. 25: 5361-5373.

Poveda, G. (2004). La hidroclimatología de Colombia: una síntesis desde la escala inter-decadal hasta la escala diurna. Rev. Acad. Colomb. Cienc. Ex. Fis. Nat. 28: 201-222.

Poveda, G. \& Mesa, O. (1999). La corriente de Chorro Superficial del Oeste ("del Chocó") y otras dos corrientes de Chorro en Colombia: climatología y variabilidad durante las fases del ENSO. Rev. Acad. Colomb. Cienc. Ex. Fis. Nat. 23: 517-528.

Poveda, G., Jaramillo, L., Vallejo, L.F. (2014). Seasonal precipitation patterns along pathways of South American lowlevel jets and aerial rivers, Water Resour. Res. 50: 98-118.

Prabha TV, Goswami BN, Murthy BS, Kulkarni JR. (2011). Nocturnal low-level jet and atmospheric streams over the rain shadow region of Indian Western Ghats. Q. J. R. Meteorol. Soc. 137: 1273-1287. Doi:10.1002/qj.818

Sakamoto, M. S., Ambrizzi, T., Poveda, G. (2012). Moisture Sources and Life Cycle of Convective Systems over Western Colombia. Advances in Meteorology, 2011: 1-11.

Sierra, J. P., Arias, P. A., Vieira, S. C., Agudelo, J. (2018). How well do CMIP5 models simulate the low-level jet in western Colombia? Climate Dynamics. 51 (5-6): 2247-2265.

Stensrud, D. (1996). Importance of Low-Level Jets to Climate: A Review. J.Climate. 9: 1698-1709.

Tedeschi, R., Cavalcanti, I., Grimm, A. (2013). Influences of two types of ENSO on South American precipitation. Int. J. Climatol. Geophys. Res. Lett. 33: 1382-1400.

Tedeschi, R. Grimm, A., Cavalcanti, I. (2016). Influence of Central and East ENSO on precipitation and its extreme events in South America during austral Autumn and Winter. Int. J. Climatol. 36: 4797-4814

Wang, C. (2007). Variability of the Caribbean Low-Level Jet and its relations to climate. Clim. Dyn. 29: 411-422.

Wilks, D. (2011). Statistical Methods in the Atmospheric Sciences. Academic Press. eBook ISBN: 9780123850232. p. 704. 